\title{
Estrategias de aislamiento de las venas pulmonares durante la ablación de fibrilación auricular
}

Mauricio Moreno $S^{1}$, Rodulfo Oyarzún $F^{1}$, Carolina Bonilla $A^{1}$, Rodrigo Tapia $C^{1}$, Edwing Martínez $R^{1}$, Ricardo Larrea $G^{1}$.

1 Unidad de Arritmias, Departamento Cardiovascular, Cliinica Dávila

\section{Resumen:}

Introducción: La ablación de la fibrilación auricular (FA) es un tratamiento potencialmente curativo para esta arritmia, siendo su principal objetivo el aislamiento o desconexión eléctrica de las venas pulmonares (VP). El aislamiento de las $4 \mathrm{VP}$ ha demostrado mayor beneficio que solo el aislamiento de la VP culpable del inicio de la FA (ablación focal). Para aislar las VP se realizan líneas de ablación alrededor de ellas, éstas se pueden efectuar en la unión veno-auricular (circunferencial antral) o en su ostium (ostial). El objetivo de este estudio es describir estas estrategias de aislamiento de las VP en un grupo de pacientes sometidos a ablación de FA.

Métodos: Los criterios de selección fueron pacientes con FA paroxística sintomática recurrente a pesar de tratamiento antiarrítmico y FA persistente sintomática sin cardiopatía estructural significativa, además, con un seguimiento mínimo de 3 meses post ablación. Se analizaron las estrategias de ablación focal versus aislamiento de las 4 VP y se evaluaron las líneas de ablación antral y ostial. El éxito fue defini- do como ausencia de FA, según síntomas y holter de arritmias de 24 horas, sin necesidad de tratamiento antiarrítmico.

Resultados: Se analizaron 50 procedimientos en 42 pacientes, por recurrencia de FA se reintervinieron 6 pacientes por una vez y un paciente 2 veces. Durante un seguimiento de $19 \pm 15$ meses se alcanzo el éxito en 30 pacientes (71.4\%). En 19 pacientes la estrategia inicial de ablación fue focal y en 23 pacientes fue en las $4 \mathrm{VP}$, alcanzándose el éxito en 12 pacientes $(63.1 \%)$ y en 20 pacientes $(87 \%)$ respectivamente $(\mathrm{p}=0.14)$. Se realizo ablación antral en las VP izquierdas en 22 casos y en las VP derechas en 14 casos, logrando la desconexión eléctrica en 3 casos (13.6\%) y ningún caso respectivamente. En los restantes casos se complemento con ablación ostial para alcanzar su aislamiento, en 4 VP este objetivo no se alcanzo. Un paciente presento una estenosis de las VP izquierdas tratada con angioplastia sin stent con buen resultado. Conclusión: La ablación de FA es una terapia efectiva para evitar la recurrencia de esta arritmia. La estrategia de ablación antral generalmente requiere de aplicaciones de radiofrecuencia adicionales a nivel del ostium de la VP para lograr su aislamiento. 


\section{Pulmonary vein isolation strategies during ablation of atrial fibrillation}

Introduction: Ablation of pulmonary veins is a potentially curative procedure for atrial fibrillation (AF). The objective is to electrically isolate the pulmonary veins from the left atrium. Ablation of all pulmonary veins (PVs) has been more effective than the isolation of the $\mathrm{PV}$ responsible for the initiation of $\mathrm{AF}$ (focal ablation). Ablation lines to isolate PVs can be performed around the PV-LA junction (circumferential) or at the PV ostium.

Aim: to describe circumferential and ostium ablation strategies in a group of patients with $\mathrm{AF}$

Methods: Patients with recurrent paroxistic AF unresponsive to anti arrhythmic therapy or patients with persistent symptomatic lone AF were included. Focal vs $4 \mathrm{PV}$ ablation as well as circumferential vs ostial techniques were compared. Success was defined as being free from $\mathrm{AF}$, as judged by symptoms and Holter recordings.

\section{Introducción}

La fibrilación auricular (FA) es la arritmia sostenida más frecuente y determina un aumento de la morbimortalidad de los pacientes que la padecen. Los antiarrítmicos tienen baja eficacia para evitar su recurrencia y a menudo se asocian a efectos adversos. La ablación de FA es un tratamiento potencialmente curativo para esta arritmia ${ }^{1}$, siendo su objetivo esencial el aislamiento o desconexión eléctrica de las venas pulmonares (VP). En algunos estudios se han reportado resultados favorables con el aislamiento de sólo la(s) VP que originaban los episodios de FA ${ }^{2}$, sin embargo otros reportes han demostrado mayor beneficio cuando se aislan todas las VP. Para aislar una VP se realiza una línea de ablación alrededor ella ${ }^{3,4}$, esta línea puede efectuarse a nivel de su ostium (ostial) o en la unión veno-auricular (antral) en forma circunferencial. El objetivo de este estudio es describir estas estrategias de aislamiento de las VP en un grupo de pacientes sometidos a ablación de FA.

\section{Métodos}

\section{Características de los pacientes}

Se incluyeron pacientes sometidos a ablación de FA en
Results: 50 procedures were performed in 42 patients. 6 patients had a repeat ablation procedure and one had 2 additional ablation procedures, due to recurrence of AF. 30 patients (71.4\%) were AF free after 19 \pm 15 months of follow-up. 12 of 19 patients $(63.1 \%)$ with focal ablation were AF free as compared to 20 of 23 submitted to $4 \mathrm{PV}$ ablation (87\%) (pNS) . Three of 22 patients (13.6\%) had successful AF ablation while none of right PVs ablation succeeded. Ostial ablation was performed after failure of circumferential ablation in unsuccessful cases. One patient developed stenosis in a left PV, which was successfully treated with stent-less angioplasty.

Conclusion: Ablation is an effective therapy to prevent recurrence of AF. Focal ablation is generally needed to achieve effective electrical isolation of PVs

Key words: Pulmonary vein isolation, Ablation, Atrail fibrillation.

Clínica Dávila, con historia de FA paroxística sintomática recurrente a pesar de tratamiento antiarrítmico y FA persistente sintomática sin cardiopatía estructural significativa; con un seguimiento mínimo de 3 meses pos ablación. Se excluyeron los pacientes con enfermedad cardíaca estructural avanzada o con contraindicación a un procedimiento invasivo. En la tabla se describen las características más relevantes de los pacientes.

\section{Tabla 1: Características de los pacientes}

\begin{tabular}{ll}
\hline Variable & $\mathrm{N} 42$ \\
\hline Sexo femenino / masculino & $6(14.3 \%)$ / $36(85.7 \%)$ \\
\hline Edad (años) & $51.8 \pm 13.5$ \\
\hline Tipo de FA paroxística / persistente & $38(90.5 \%) / 4(9.5 \%)$ \\
\hline Tiempo de evolución (meses) & $67.6 \pm 61.2$ \\
Antiarrítmicos (n) & $1.13 \pm 0.35$ \\
Hipertensión arterial & $16(38 \%)$ \\
Diabetes Mellitas & $2(4.7 \%)$ \\
Cardiopatía coronaria & $4(9.5 \%)$ \\
Fracción de eyección & $65.7 \% \pm 5$ \\
Diámetro de Al (mm) & $37.6 \pm 5.4$
\end{tabular}




\section{Estudio electrofisiológico}

El procedimiento se realizó bajo sedación con infusión continua de propofol. Se realizaron 3 punciones venosas femorales derechas para avanzar un catéter decapolar al seno coronario, un catéter laso para registrar la actividad eléctrica de las VPs y un catéter de ablación irrigado abierto (Sprinkler, Medtronic). Para avanzar los catéteres a la aurícula izquierda (AI) se efectuó una punción transeptal guiada por radioscopía. Después del acceso transeptal se administro heparina endovenosa para mantener un tiempo de coagulación activado alrededor de 300 segundos, con mediciones cada 20 a 30 minutos. Luego se realizó angiografía de las VP. Para registrar las señales eléctricas intracardiacas se utilizó un polígrafo EP-TRACER (CardioTek). En la mayoría de los pacientes se construyó un mapa anatómico tridimensional con el sistema de navegación EnSITE-NAVx (St Jude Medical). Este mapa resultó de la fusión entre la información eléctrica del catéter utilizado (de ablación y laso) y la imagen de la AI y VP por tomografía multicorte realizada previamente.

\section{Ablación}

Los parámetros de ablación con radiofrecuencia (RF) con catéter irrigado fueron temperatura $45^{\circ} \mathrm{C}$ y potencia 30 watts. Los potenciales dentro de la VP fueron registrados con un catéter laso. El aislamiento de cada VP consistió en el bloqueo eléctrico bidireccional a la AI, lo cual se estableció cuando desaparecieron o se disociaron los potenciales de VP (ver fig. 1) y no hubo conducción a la AI al estimular dentro de la VP.

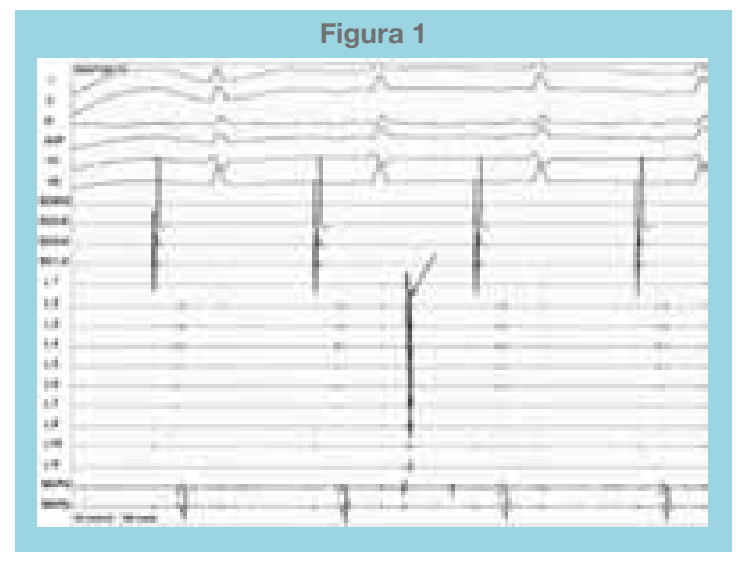

. Desconexion electrica de la VP superior derecha. En el registro del cateter laso se observa una ectopia de la VP superior derecha no conducida a AI (flecha). SC: seno coronario, L: laso, MAPd: cateter de ablacion distal, MAPp: cateter de ablacion proximal.

Inicialmente en nuestro laboratorio la estrategia de ablación fue focal, es decir, se aisló la VP con mayor probabilidad de ser culpable del inicio de la FA, para lo cual se determinó la VP donde se originaban las ectopias auriculares espontáneas o inducidas con adenosina y/o isoproterenol. Posteriormente, la estrategia evolucionó al aislamiento empírico de todas las VP que presentaban conducción eléctrica.

La ablación ostial consistió en aplicaciones de RF en el ostium de la VP involucrando todo su perímetro o parte de él (segmentaria). La ablación antral consistió en aplicaciones de RF en la unión venoatrial de VP ipsilaterales creando líneas circunferenciales alrededor de ellas. Esto fue guiado por un mapa anatómico tridimensional (ver fig. 2). En ambos protocolos el objetivo fue siempre la desconexión eléctrica de la(s) VP. En los casos en que no se lograron aislar las VP mediante ablación astral, se realizaron aplicaciones adicionales de RF ostiales. Aquellas VP que no mostraron conducción eléctrica no fueron ablacionadas.

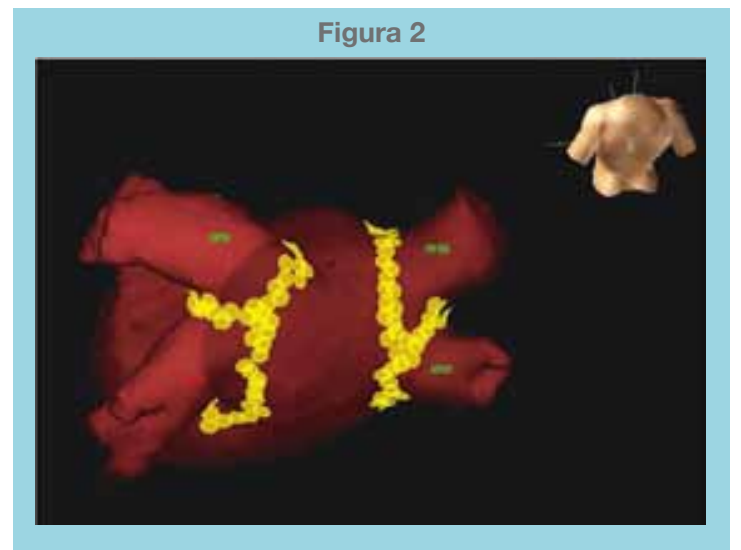

Ablacion antral de las 4 VPs. Mapa electroanatomico de AI y VP construido con el sistema EnSITE donde se registran las lineas de ablacion circunferenciales alrededor de las VP.

Posterior al procedimiento los pacientes quedaron en la unidad coronaria y a las 6 horas se administró clexane subcutáneo en dosis $50 \%$ de la terapéutica. Al día siguiente del procedimiento se realizó un ecocardiograma transtorácico de control. Se indicó un antiarrítmico por 1 mes y a los 3 meses se evaluó la suspensión del anticoagulante según el riesgo tromboembólico. Para evaluar la recurrencia de FA los pacientes fueron seguidos clínicamente y con holter de arritmias de 24 horas dentro de los 3 meses pos ablación, al sexto mes y al año.

Se definió como éxito de la ablación de FA cuando el paciente permaneció clínicamente y en el holter libre de FA sin fármacos antiarrítmicos. Se compararon los resultados entre ablación de todas las VP y ablación focal, y se analizó la ablación antral y ostial.

\section{Análisis estadístico}

Las variables continuas se expresan con media y una desviación estándar. Se utilizó chi cuadrado o test exacto de 
Fisher cuando estaba indicado para analizar las variables dicotómicas. Se consideró significativo un valor de $\mathrm{p}$ de 0.05 .

\section{Resultados}

Se realizaron 50 procedimientos de ablación de FA en 42 pacientes. En algunos pacientes con recurrencia pos ablación se repitió el procedimiento una vez (6 pacientes) y 2 veces en un paciente, esto determinó una proporción de reintervención de 19\%. Durante un tiempo de seguimiento promedio de $19 \pm 15$ meses se observaron 30 pacientes libres de FA sin antiarrítmicos $(71.4 \%)$.

De 4 pacientes con FA persistente $(9.5 \%) 2$ permanecen sin arritmia (50\%), mientras que los 2 casos con recurrencia están con tratamiento farmacológico para control de frecuencia cardíaca. De los 38 pacientes con FA paroxística están libres de FA 28 pacientes $(73.6 \%)$.

En 19 pacientes, la primera estrategia de ablación fue focal y permanecen libres de FA 12 pacientes $(63.1 \%)$ En 23 pacientes la primera estrategia de ablación fue el aislamiento de las $4 \mathrm{VP}$ y permanecen libres de FA 18 pacientes $(78.2 \%)$. No se observó una diferencia estadísticamente significativa entre ambas estrategias $(\mathrm{p}=0.14)$. $\mathrm{Al}$ inicio del procedimiento 11 casos estaban en FA (22\%), incluyendo los 4 casos de FA persistente. Un caso pasó a ritmo sinusal durante la ablación, en 3 casos se realizó cardioversión eléctrica inmediatamente antes del procedimiento y en 7 casos la cardioversión fue durante el procedimiento. Se observaron potenciales de VP en 163 de las 168 VP evaluadas (97\%). Las VP sin actividad eléctrica correspondieron a la VP inferior izquierda 1 caso $(0.59 \%)$, VP superior derecha 2 casos $(1.2 \%)$ y VP inferior derecha 2 casos $(1.2 \%)$.

La estrategia de ablación inicial en las VP izquierdas fue circunferencial antral en 22 casos, mediante la cual se logró la desconexión eléctrica de la VP superior izquierda en 5 casos (22.7\%), VP inferior izquierda en 6 casos (27.2\%) y ambas VPen 3 casos (13.6\%). La estrategia de ablación inicial en las VP derechas fue circunferencial antral en 14 casos, mediante la cual se logró la desconexión eléctrica en la VP superior derecha en 1 caso $(7.1 \%)$, VP inferior derecha en 3 casos $(21.4 \%)$ y en ningún caso ambas VP. Cuando con la ablación circunferencial no se aislaron las VP se complemento con aplicaciones de RF a nivel ostial hasta la desconexión eléctrica de la VP. En 4 VP no se logró la desconexión eléctrica: 1 VP superior izquierda, 1 VP inferior izquierda, 1 VP superior derecha y 1 VP inferior derecha.

Un paciente presentó estenosis de las VP izquierdas, manifestándose con hemoptisis; se diagnosticó con TAC multicorte y luego se confirmó con angiografía de VP. Se realizó angioplastía sin stent en las VP con buen resultado inmediato y en el control angiográfíco a los 4 meses.

\section{Discusión}

Según los datos reportados el éxito de la ablación de FA a un año, incluidas las reintervenciones, varía entre 70 a $85 \%$, sin embargo estos resultados pueden disminuir cuando se incluyen pacientes con FA crónica, alteraciones estructurales cardíacas importantes y según la experiencia del centro ${ }^{5,6}$. En este estudio se observó un éxito del $71 \%$, incluyendo los pacientes reintervenidos (19\%), lo cual es comparable con la información publicada.

Las estrategias de ablación descritas en este estudio representan la evolución de la técnica en nuestro centro y se observó una tendencia a mayor éxito con el aislamiento de las $4 \mathrm{VP}$, que el aislamiento de la VP, que probablemente originaba la FA. La transición de la estrategia de aislamiento focal a total (4 VP) en nuestro centro se relacionó con la incorporación de un sistema de navegación tridimensional que nos permitió establecer mayor seguridad para el paciente y disminuir los tiempos de exposición radiológica asociados a un procedimiento más complejo. Aunque hay datos favorables sobre ablación focal el seguimiento a largo plazo muestra una reducción de este beneficio inicial ${ }^{2}$; consecuentemente los resultados de este estudio muestran una tendencia favorable a favor de la ablación de las 4 VP. Cuando se realizó una ablación circunferencial antral, se logró aislar ambas VP izquierdas en el 13\% y en ningún caso ambas VP derechas. Por ello, generalmente fue necesario realizar más aplicaciones de RF a nivel ostial hasta alcanzar la desconexión eléctrica. En la práctica, cuando se realizan líneas de ablación circunferenciales alrededor de las VP, alejadas del ostium, creamos frentes de ablación más amplios con mayor probabilidad de tener gaps que pueden mantener la conexión eléctrica entre la VPy la AI. De hecho uno de los factores más importantes para lograr el adecuado aislamiento es la creación de líneas continuas de ablación. Además, la creación de líneas discontinuas puede ser proarrítmica. Las variables que influyen en esto son el contacto del catéter de ablación con el endocardio, buena orientación anatómica tridimensional, dificultades anatómicas particulares de cada paciente, experiencia de los operadores, etc.

En favor de la ablación circunferencial antral esta la reducción del riesgo de estenosis de VP y, además, que probablemente se eliminan focos de mantención de FA al afectar áreas de reentrada como rotores y producir modu- 
lación autonómica sobre ganglios parasimpáticos locales 7. Según nuestros datos la ablación circunferencial mostró una baja eficacia para aislar las VPy fue casi de regla necesario efectuar más aplicaciones de RF a nivel ostial. Entonces el procedimiento se hace más demandante porque aumentamos el área de ablación debido a estos dos flancos: antral y ostial; y cambiamos un procedimiento donde se aplica RF según criterios anatómicos a criterios electrofisiológicos. Por ello, hoy en día se realizan esfuerzos para desarrollar nuevas tecnologías que permitan crear líneas de ablación más efectivas mediante un mejor contacto del catéter con la aurícula.

En algunas ocasiones no se logra eliminar los potenciales de VP a pesar de múltiples aplicaciones de RF antral y/o ostial, en estos casos siempre es necesario descartar los potenciales lejanos; como por ejemplo, potenciales de orejuela izquierda en la VP superior izquierda. Ahora en este estudio en el $2.3 \%$ de las VP no se logró la desco- nexión eléctrica, esto podría explicarse por la persistencia de un gap inadvertido entre la VP y la AI, presencia de una conexión eléctrica entre VP ipsilaterales y conexiones epicárdicas entre VPy AI ${ }^{8}$.

Una limitación de este estudio es el seguimiento de los pacientes, debido a un probable insuficiente diagnóstico de episodios de FA ya que pueden ser asintomáticos ${ }^{9} \mathrm{y}$ no ser registrados en el holter de 24 horas. Aunque no se observaron diferencias estadísticamente significativas entre las estrategias de ablación, si hubieron tendencias que podrían confirmarse en un estudio con mayor número de pacientes.

En conclusión, en este estudio se observó que en la mayoría de los casos de ablación circunferencial antral fue necesario complementar con aplicaciones de RF ostial. Según estos resultados la ablación de FA es una alternativa terapéutica efectiva para pacientes con FA sintomática refractaria a terapia antiarrítmica.

\section{Referencias:}

1. HAÏSSAGUERRE M, JAIMLIS P, SHAH DC, TAKAHASHI

A, HOCINI M, QUINIOU G, et al. Spontaneous initiation of atrial fibrillation by ectopic beats originating in the pulmonary veins. N Engl J Med. 1998; 339: 659-666.

2. HAISSAGURRE M, JAIS P, SHAH D, GARRIGUE S, TAKAHASHI A, LAVERGNE T, et al.. Electrophysiological end point for catheter ablation of atrial fibrillation initiated from multiple pulmonary venous foci. Circulation 2000; 101: 1409 $-1417$.

3. ORAL H, SCHARF C, CHUGH A, HALL B, CHEUNG P, GOOD E, et al. Segmental pulmonary vein ostial ablation versus left atrial ablation. Circulation. 2003; 108: 2355 - 2360.

4. OUYANG F, BÄNSCH D, ERNST S, SCHAUMANN A, HACHIYA H, CHEN M, et al. Complete isolation of the left atrium surrounding the pulmonary veins: new insights from the double Lasso technique in paroxysmal atrial fibrillation. Circulation. 2004; 110: 2960-2968.

5. JALIS P, CAUCHEMEZ B, MACLE L, DAOUD E, KHAIRY

$\mathrm{P}, \mathrm{SUBBIAH} \mathrm{R}$, et al. Catheter ablation versus antiarrhythmic drugs for atrial fibrillation: the A4 study. Circulation. 2008; 118: $2498-2505$.

6. CALKINS H, REYNOLDS MR, SPECTOR P, SONDHI M, XU Y, MARTIN A, et al. Treatment of atrial fibrillation with antiarrhythmic drugs or radiofrequency ablation: two systematic literature reviews and meta-analyses. Circ Arrhythm Electrophysiol. 2009; 2 : 349-361.

7. BAUER A, DEINSENHOFER I, SCHNEIDER R. Effects of circumferential or segmental pulmonary vein ablation for paroxysmal atrial fibrillation on cardiac autonomic function. Heart Rhythm 2006; 3: 1428 - 1435.

8. TAKAHASHI A, IESAKA Y, TAKAHASHI Y, TAKAHASHI R, KOBAHASHI K, TAKAGI K, et al. Electrical connections between pulmonary veins: implication for ostial ablation of pulmonary veins in patients with paroxysmal atrial fibrillation. Circulation. 2002; 105: 2998 - 3003.

9. HINDRICKS G, PIORKOWSKI C, TANNER H, KOBZA R, GERDS-LI J, CARBUCIOCCHIO C, et al. Perception of atrial fibrillation before and after radiofrequency catheter ablation: relevance of asymptomatic arrhythmia recurrence. Circulation. 2005; 112: 307 - 313 . 